\title{
Laser and brachytherapy in the palliation of adenocarcinoma of the oesophagus and cardia
}

\author{
G M Spencer, S M Thorpe, I R Sargeant, G M Blackman, J Solano, J S Tobias, S G Bown
}

\begin{abstract}
Background-Palliation of malignant dysphagia is possible by a variety of methods although all have significant drawbacks. Laser therapy is an effective and safe treatment but has to be repeated at four to five weekly intervals to maintain palliation. A means of augmenting the benefits while reducing the need for repeat treatments would be highly beneficial to these patients.
\end{abstract}

Aims-To prospectively explore the safety and efficacy of intraluminal radiotherapy (brachytherapy) when used to augment laser recanalisation for malignant dysphagia.

Patients-Nineteen patients with dysphagia due to advanced adenocarcinoma of the oesophagus or cardia were recruited.

Methods-All patients received laser recanalisation until able to swallow a soft diet or better, before the application of a single dose of brachytherapy $(10 \mathrm{~Gy}$ at $1 \mathrm{~cm}$ from the source). Patients were followed up and treated promptly by further endoscopic means in the event of their dysphagia worsening.

Results-Six patients $(32 \%)$ required no further treatment until death at a median of 10 weeks (range 1-20 weeks). Further therapy was required at a median of 11 weeks (range 4-37 weeks) after brachytherapy for those 13 patients with recurrent dysphagia. Subsequent symptom control required endoscopic intervention at an average of once every nine weeks. There was no mortality associated with laser or brachytherapy. Median survival from initial treatment and including the one survivor was 36 weeks (range 5-132 weeks).

Conclusions-Laser plus brachytherapy offers a safe and effective means of palliating malignant dysphagia due to adenocarcinoma, with a longer dysphagia free interval than historical controls treated with laser alone.

(Gut 1996; 39: 726-731)

Keywords: oesophagus, gastric cardia, adenocarcinoma, laser, brachytherapy.

The incidence of adenocarcinoma of the oesophagus and gastric cardia is increasing in the United Kingdom. ${ }^{1}$ Unfortunately over $90 \%$ of tumours have penetrated the oesophageal wall at presentation with $72 \%$ having spread to involve the lymph nodes even in those lesions that are deemed resectable. ${ }^{2}$ The disease principally affects an elderly population, with more than $60 \%$ of patients having no prospect of cure because of advanced disease or intercurrent illness precluding surgery or radical radiotherapy. ${ }^{34}$ Computed tomography (CT) imaging and endoscopic ultrasound have improved our ability to stage tumours of the upper gastrointestinal tract more effectively. The decision, therefore, actively to pursue palliation from the outset may now be made with more accuracy and conviction, rather than embarking on a futile attempt at radical therapy.

Endoscopic methods of palliation have become an accepted form of treatment for those patients who are not amenable to radical radiotherapy or an attempt at surgical cure. Laser therapy has an excellent safety profile and is highly efficacious at relieving dysphagia, ${ }^{5}$ although initial recanalisation and dysphagia relief often requires several treatments in short succession. Unfortunately to maintain palliation, laser therapy has then to be repeated at four to six week intervals. Radiotherapeutic techniques have been used to augment and prolong the laser effect, ${ }^{6}$ but while external beam radiotherapy prolongs the interval between laser sessions, its application requires that the palliative dose is given in several fractions over one to two weeks to minimise toxicity. The demands on the patient are thus little improved by this 'adjuvant' treatment. In contrast with external beam radiotherapy, however, intraluminal radiotherapy (brachytherapy) allows the application of a therapeutic dose of radiotherapy in a single fraction. This has an obvious attraction in terms of palliation. Sander $e t a l^{7}$ have suggested, however, that this form of treatment, while useful for palliating squamous tumours, was detrimental to patients with adenocarcinoma. Our aim was to further explore the usefulness of single fraction brachytherapy for adenocarcinomas, as an adjunct to laser recanalisation, with a prospective trial.

\section{Methods}

Patients with confirmed adenocarcinoma of the oesophagus or gastric cardia were recruited between July 1992 and June 1994. All patients had been deemed only suitable for palliative therapy owing to advanced primary disease, metastatic spread, cardiopulmonary disease or general frailty, and ill health. Patients treated with chemotherapy, radiotherapy or the insertion of an endoprosthesis were excluded from the trial. Patients with malignancies 
TABLE I Dysphagia grading

\begin{tabular}{ll}
\hline Quality of swallowing & Grade \\
\hline Normal diet & 0 \\
Some solids & 1 \\
Soft diet & 2 \\
Liquids only & 3 \\
Dysphagia to liquids & 4 \\
\hline
\end{tabular}

TABLE II Patient characteristics

\begin{tabular}{ll}
\hline Total number of patients & 19 \\
Male/female & $12 / 7$ \\
Age (median) & 79 (range 62-92) \\
Tumour site: & \\
$\quad$ oesophagus & 6 \\
$\quad$ cardia & 13 \\
Tumour length (median)/cm & 5 (range 3-10) \\
$\begin{array}{l}\text { Distance of top of tumour from } \\
\text { incisors (median)/cm }\end{array}$ & 32 (range 26-45) \\
$\begin{array}{l}\text { Dysphagia grade at } \\
\text { presentation (median) }\end{array}$ & 3 (range 1-4) \\
$\begin{array}{c}\text { Dysphagia grade before } \\
\text { brachytherapy (median) }\end{array}$ & 2 (range 0-2) \\
$\begin{array}{c}\text { Dilatation required during } \\
\text { initial recanalisation }\end{array}$ & $10 / 19$ \\
\hline
\end{tabular}

arising in other organs and causing dysphagia by direct or metastatic spread were also excluded. All patients had dysphagia as a result of predominantly exophytic tumours. Dysphagia was scored as shown in Table I. Those patients with predominantly extrinsic compression of the oesophagus were excluded from this investigation. Patient details are shown in Table II

Patients were treated with lignocaine local anaesthetic throat spray and sedated with a combination of intravenous pethidine plus diazemuls (Dumex, Princes Risborough, UK) or midazolam (Hypnovel, Roche, Welwyn Garden City, UK) for all endoscopic procedures. Where the passage of the laser endoscope (Olympus 1T20, Keymed Ltd, Southend on Sea, UK) was made difficult owing to tumour narrowing of the oesophageal lumen, dilatation was performed using 12 and $18 \mathrm{~mm}$ Celestin dilators (Medoc, Tetbury, UK) under fluoroscopic control. Unfortunately, coating of both normal mucosa and tumour with fresh blood and clot often impaired endoscopic vision immediately after dilatation. In view of the potential risk of exacerbating a minor obscured tear by applying laser immediately, laser therapy was usually applied the following day, or as soon as possible thereafter, by which time views had improved considerably. Although the laser is often used to provide haemostasis for bleeding tumours, it is inappropriate to use it for that purpose here, as the oozing is due to the trauma of the dilatation and almost always settles spontaneously. Laser energy was provided by a Neodymium:Yttrium Aluminium Garnet (Nd:YAG) laser (Flexilase, Living Technology, Glasgow, UK) using powers from $60-80 \mathrm{~W}$. All endoscopic therapy was performed by GMS, SGB or IRS at University College London Hospitals. Laser therapy was applied at intervals of a few days, until the patient was able to swallow a soft diet (grade 2) or better.
To assist brachytherapy planning, a final endoscopy was performed to place external skin markers (paper clips) at the level of the proximal and distal limits of the tumour using fluoroscopy. A 12 or 14 French Ryles nasogastric tube was then passed into the stomach and the position confirmed with fluoroscopy. The patient was transferred immediately to the Microselectron (Nucleotron UK Ltd, Tattenhall, UK) facility where a limited planning barium swallow was performed, to confirm the tumour limits. Brachytherapy was applied to the length of the tumour plus a $2 \mathrm{~cm}$ margin at each end, using a high dose rate Iridium-192 (HDR Ir-192) wire applying $10 \mathrm{~Gy}$ at $1 \mathrm{~cm}$ from the source. The nasogastric tube was removed immediately after treatment and the patient allowed home as soon as practical.

The majority of laser and brachytherapy treatments were applied on an outpatient basis. Subsequent treatment was only given when swallowing deteriorated to the extent that the patient experienced difficulty with a soft diet, whereupon laser, dilatation or intubation was used according to clinical need. Laser was reapplied to exophytic tumours, dilatation where strictures were predominantly smooth and fibrous due to brachytherapy scarring, with intubation reserved for lesions that had become predominantly extrinsic or where the patient's condition had deteriorated and repeated laser sessions or dilatations were felt unjustified. Intubation, where necessary, was performed using the Celestin tube (Medoc, Tetbury, UK). Efficacy of treatment was judged on the dysphagia scoring, complications, and side effects encountered. Patients were contacted weekly by telephone and interviewed by the author (GMS) or research sister (ST). The interval between brachytherapy and further treatment was deemed the dysphagia free interval.

Informed consent was obtained from all those patients entered in the trial.

\section{Results}

Nineteen patients (12 men, seven women; median age 79 years) were recruited into this study. The lesion was deemed incurable on the grounds of local disease stage in nine, verified metastatic disease in two, recurrent disease after previous surgery in one, valvular and ischaemic heart disease in two, and general frailty and old age in the remaining five patients (Table III). Ten of 19 patients required dilatation, in addition to laser therapy, as part of their initial recanalisation.

\section{TABLE III Reason for palliation}

\begin{tabular}{ll}
\hline Reason for palliation & Number (\%) \\
\hline Advanced local disease at: & \\
computed tomography & $4(21)$ \\
endoscopic ultrasound & $4(21)$ \\
laparotomy & $1(5)$ \\
Liver metastases & $2(11)$ \\
Recurrent disease after surgery & $1(5)$ \\
Valvular/ischaemic heart disease & $2(11)$ \\
General frailty/old age & $5(26)$ \\
\hline
\end{tabular}




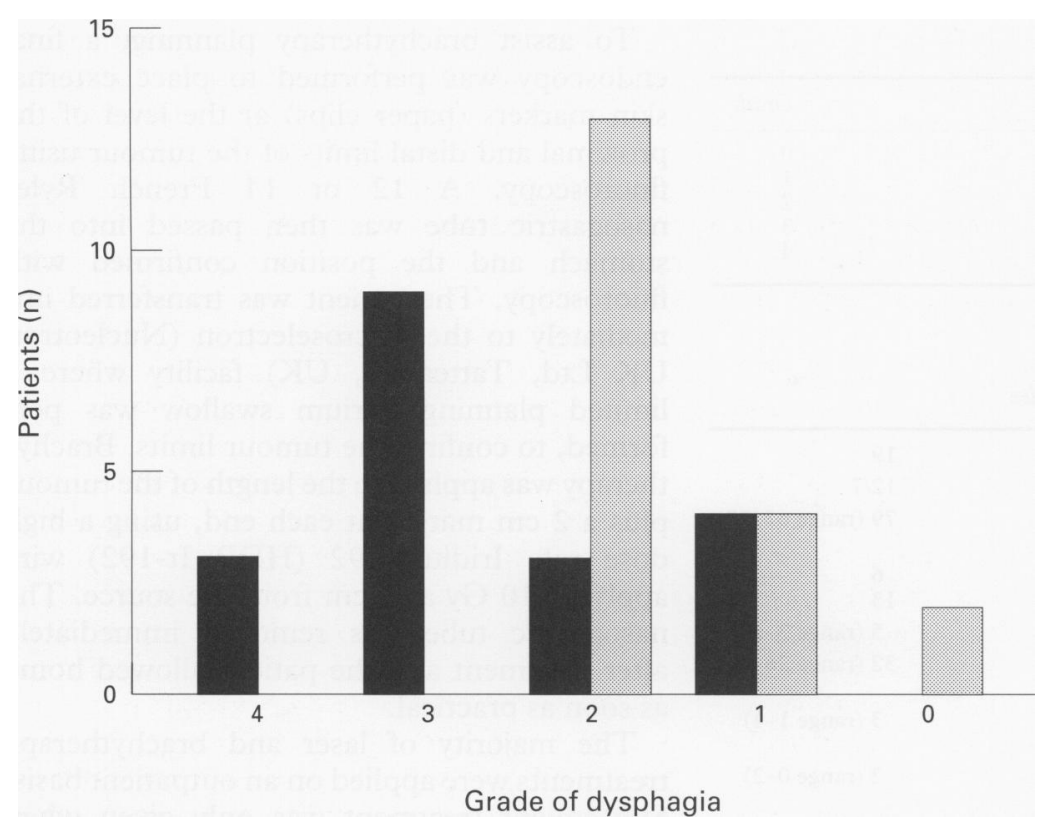

Figure 1: Improvement in dysphagia as a result of laser treatment (19 patients). Solid bars represent dysphagia grade prior to laser therapy. Shaded bars represent dysphagia grade after laser but before brachytherapy.

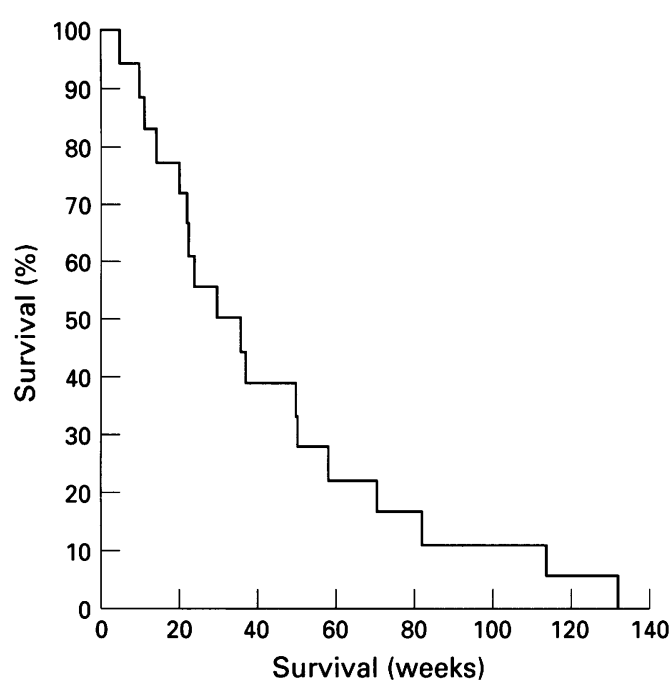

Figure 3: Percentage survival from first treatment in weeks ( $n=18)$ excluding the one survivor.

recurrent symptoms requiring further endoscopic therapy, dilatation was required for 10 $(77 \%)$, laser for nine $(69 \%)$, and Celestin tube insertion for six (46\%), three of whom required subsequent tube adjustments. In those patients returning for treatment for recurrent dysphagia, further endoscopic treatment was required at a mean of nine week intervals, until death or time of writing with respect to the one survivor.

Including the survivor, median survival from first treatment was 36 weeks (mean 47 weeks, range 5-132 weeks) (Fig 3). The remaining patient is swallowing a soft diet at 97 weeks.

Two patients underwent a second brachytherapy session for recurrent dysphagia at 40 and 49 weeks after the first. They underwent further laser recanalisation immediately followed by brachytherapy applying a further 5 Gy at $1 \mathrm{~cm}$. Dysphagia relief lasted for another 11 weeks before both patients required dilatation for fibrous stricturing. Radiation oesophagitis occurred as a result of this second dose of radiotherapy in both patients, lasting for four and 11 weeks and was associated with oesophageal ulceration in the latter. Both patients however experienced pain only during swallowing and were not restricted in their daily activities.

Fifteen patients required a mean lifetime total of 17 days in hospital (median 13 days, range 2-68 days) from their initial treatment until death, or follow up for the survivor. Four patients did not require any period of inpatient treatment, but were able to attend as day cases for endoscopic therapy throughout the duration of their illness. Day case treatment was used by a total of 15 patients, on a mean of six occasions (median 5, range 1-15). Eighty eight of a total of 153 treatments $(58 \%)$ were provided as day cases.

There was no mortality associated with laser, dilatation, or brachytherapy. No laser associated morbidity was seen. Brachytherapy induced radiation oesophagitis caused mildly painful swallowing in six (32\%) patients and lasted 1-11 weeks but was not seriously 
troublesome. There was rarely any discomfort other than when swallowing. This complication did not deter two patients from receiving a second 5 Gy brachytherapy treatment as they felt that the dysphagia relief justified the shortterm discomfort. Symptoms responded to treatment with combinations of analgesic, antacid, omeprazole, and sucralfate suspension. There were no dilatation associated complications but Celestin tube insertion caused perforation into the mediastinum in one case. The perforation was seen proximal to the Celestin tube. To protect the defect, a cuffed Wilson Cook tube (Wilson Cook Europe Limited) was inserted above the Celestin tube. A barium swallow confirmed that the defect was covered. The patient tolerated a liquidised diet and was able to return home until his death five weeks later. The remaining five patients were able to tolerate a soft diet after Celestin tube insertion. Four of six patients treated with Celestin tubes developed problems due to tube migration/blockage. Three responded to endoscopic adjustment.

\section{Discussion}

The aim of palliation is to maintain the patients' independence and ensure a good quality of life, with as few interventions and procedure related complications as possible, for the remainder of their lives. In patients with oesophageal and cardia cancer, the principal symptom requiring palliation is dysphagia. All too often palliative techniques have offered suboptimal symptom control, repeated interventions or were associated with either long periods of hospitalisation or high rates of morbidity and mortality.

External beam radiotherapy (XRT) is commonly used to palliate oesophagogastric cancers. Unfortunately, the large fields involved in treating advanced lesions preclude the use of larger doses, despite fractionation, for fears of toxicity. In addition, treatment compliance becomes more of a problem with large doses and dysphagia relief may take several weeks. ${ }^{8}$ In some cases dysphagia is transiently exacerbated by XRT before ultimate improvement. Caspers et $a l^{9}$ have shown that only half of all patients palliated with XRT will achieve benefit for their remaining life with those who swallow poorly at presentation deriving little benefit from either radical or palliative XRT monotherapy when compared with those who swallow well.

Laser therapy offers initial relief in approximately $90 \%$ of patients experiencing dysphagia due to oesophageal and cardia cancers. ${ }^{10}$ Unfortunately, treatment has to be repeated at four to six weekly intervals to maintain symptom control. In this way $85 \%$ of patients achieve long term relief. ${ }^{11}$ The cost to the patient is one of multiple hospital visits, multiple endoscopy sessions and often a short inpatient stay if the patient is frail or has to travel far to tertiary referral centres. This inconvenience has been considered worthwhile in view of the excellent safety profile of laser therapy with its associated morbidity of $4 \%$ and mortality of under $1 \% .{ }^{12}$ In addition, one third of patients would expect to swallow a normal diet after laser therapy, with approximately $60 \%$ managing a soft diet. Ten per cent would tolerate fluids only and would be classed as laser treatment failures, being offered an alternative treatment modality. It has been established that quality of life is strongly associated with the severity of dysphagia ${ }^{13}$ and therefore techniques aimed at relieving dysphagia should lead to an improvement in the quality of life.

When brachytherapy is used without the laser, endoscopic intervention is often necessary to dilate the neoplastic stricture before placement of the source applicator device. It may also be required for the management of treatment failures and recurrent dysphagia, due to fibrous stricturing or tumour regrowth, so the addition of laser therapy is not necessarily a major extra burden for patients. Brachytherapy when applied as a monotherapy has been shown to offer palliation for oesophageal cancer, improving swallowing in $65 \%$ of patients treated and lasting for a median duration of 15 weeks. ${ }^{14}$ The quality of dysphagia relief was not however quantified. A prospective randomised study comparing brachytherapy with laser treatment revealed similar initial results for both modalities. ${ }^{15}$ Initial improvement in dysphagia scores occurred in $91 \%$ receiving laser and $83 \%$ receiving brachytherapy. This fell to $81 \%$ and $75 \%$ respectively at two months. Performance scores improved in approximately one third of patients regardless of treatment modality. Sixty six per cent of patients treated with laser and $60 \%$ of those treated with brachytherapy were able to tolerate a semisolid diet at death or six month follow up. Retreatment was more common in the laser group, although treatment failures - that is, a failure to relieve dysphagia initially, were identical.

In an early study combining the two, laser recanalisation and brachytherapy together permanently relieved dysphagia in $77 \%$ of patients undergoing palliation for either squamous or adenocarcinomas of the oesophagus, cardia and at sites of anastomotic recurrence. ${ }^{16}$ Sixteen of 48 patients treated had died at the time of publication with a mean survival of 5.9 months, but no data were given on dysphagia grades. Sander et $a l^{7}{ }^{7}$ in a randomised prospective trial of laser only versus laser plus brachytherapy, found that laser plus brachytherapy doubled the dysphagia free first interval from 30 to 65 days in patients with squamous carcinoma. Those with adenocarcinoma fared less well, and required more frequent endoscopic procedures. Sander suggested, on the basis of this finding and a slightly shorter survival, that supplementary brachytherapy was detrimental in this small subgroup. Recurrent dysphagia developed in all patients living longer than a few weeks or those not having already required intubation for oesophagotracheal fistulae, that is, $72 \%$ of patients treated. Our study indicates that brachytherapy following initial laser recanalisation was not detrimental to the patients' well 
being. Indeed the dysphagia free interval was doubled to 10-11 weeks when compared with historical controls receiving laser only, where dysphagia is typically relieved for four to six weeks. Recurrent dysphagia was however seen in all those patients living longer than a few months.

Laser and brachytherapy must be compared with other endoscopic modalities. Intralesion injection of ethanol has been used to recanalise oesophagogastric tumours. ${ }^{17}$ This modality has many of the benefits of laser therapy, at a fraction of the cost, but is less precise and still has to be repeated at four weekly intervals to maintain dysphagia relief. The distribution of ethanol when instilled into the tumour is unpredictable and may be quite difficult to control when the tumour is hard and scirrhous in nature. The addition of any form of radiotherapy to augment this form of recanalisation has not been explored.

The placement of silicon rubber tubes to palliate oesophageal cancer predates laser therapy and is still the treatment of first choice in many centres in the UK. Unfortunately, fewer than $10 \%$ of patients swallow normally, the majority just tolerating a soft diet. ${ }^{18} 19$ As many as $30 \%$ will only manage liquids. In addition, tube insertion has a mortality of $3-12 \%{ }^{20}$ Problems due to tube migration, tumour overgrowth, late perforation, and bolus obstruction are seen in up to $44 \%$ of patients. Tube insertion was historically viewed as a 'one off procedure, although we now know that almost half of all patients treated in this manner will have at least one further endoscopy in their remaining life. ${ }^{21}$ Many would consider intubation as complementary to laser, in situations like extrinsic compression or failure of laser palliation. Further, many patients present in the twilight of their illness and it is inappropriate to pursue laser palliation when all that is required is the amelioration of dysphagia to liquids. The anorexia of advanced malignant disease negates any benefit from better swallowing.

The only prospective studies comparing quality of life with endoprostheses and laser therapy failed to show any significant advantage for either. Both led to an improvement in the quality of life until the last few weeks of the illness. ${ }^{1321}$

The usefulness of expansile metal stents in the palliation of oesophageal carcinoma is not yet clear owing to the continuing evolution of these devices and the limited data currently available. They are also 10 times the cost of conventional tubes. The relative ease of insertion, requiring at most a $12-15 \mathrm{~mm}$ dilatation contrasts with the procedure involved with conventional rubber tube placement, where up to an $18 \mathrm{~mm}$ dilatation is mandatory. This difference alone probably accounts for the lesser mortality of metal stent insertion when compared with silicon rubber endoprosthesis. Despite this, metal stent insertion may be associated with a morbidity of as high as $17 \%$ and mortality of up to $8 \%,{ }^{22}$ although lesser values may become the norm as experience increases. The frequency of recurrent dys- phagia is similar for both conventional and metal stents. Knyrim et $a l^{23}$ reported $33 \%$ of patients with each type experiencing problems, in the longer term, due to bolus obstruction, tumour overgrowth, tumour ingrowth (metal stents only), prosthesis migration or tracheooesophageal fistula. Grund et $a l^{24}$ found that re-intervention was necessary in $67 \%$ of 114 patients treated with metal stents, mainly as a result of tumour ingrowth. An average of 3.5 endoscopic treatments were required per patient during their lifetime including preparatory therapy before stent placement.

These studies suggest that despite initial hopes, expanding metal stents may only offer reasonable palliation when supported by further endoscopic intervention as is the case with laser therapy and rubber tube insertion. Initial studies concerning covered metal stents suggest that while they are free of ingrowth problems, they have the disadvantage of stent migration in up to $10 \%$ of patients. ${ }^{25}$

It is clear that no single treatment modality offers a complete solution to the variety of problems associated with palliating malignant dysphagia. Complementary treatments of confirmed efficacy and patient acceptability are likely to be the way forward. This will demand a degree of expertise and flexibility in terms of the therapeutic options a practitioner has to offer. Further randomised studies are necessary to explore these options while taking into account cost and patient quality of life. A prospective randomised trial is in progress to define more closely the role of brachytherapy when used to augment laser palliation for adenocarcinoma of the oesophagus and cardia.

1 Cheng KK, Day NE. Oesophageal cancer in Britain (letter). $B M F$ 1992; 304: 711 .

2 Watson A. Therapeutic options and patient selection in the management of oesophageal carcinoma. In: Watson A, Celestin L R, eds. Disorders of the oesophagus. London: Pitman, 1984: 167-86.

3 Watson A. Surgery for carcinoma of the oesophagus. Postgrad Med $\mathcal{F} 1988 ; 64: 860-4$.

4 Desa L, Raghunath AS, Chawla SL, Peel AL, Dellipiani AW. Treatment policy for management of carcinoma of the oesophagus. $B r f$ Surg 1988; 75: 275-8.

5 Maunour V, Brunetaud JM, Cochelard P. Endoscopic palliation for inoperable malignant dysphagia: long term follow up. Gut 1992; 33: 1602-7.

6 Sargeant IR, Loizou LA, Tobias JS, Blackman G, Thorpe S, Bown SG. Radiation enhancement of laser palliation for malignant dysphagia: a pilot study. Gut 1992; 33: 1597-601.

7 Sander R, Hagenmueller F, Sander C, Riess G, Classen M. Laser versus laser plus afterloading with iridium-192 in the palliative treatment of malignant stenosis of the the palliative treatment of malignant stenosis of the esophagus: a prospective, randomised and

8 Pearson JG. Present status and future potential of radiotherapy in the management of oesophageal cancer. In: Silber F, ed. Carcinoma of the oesophagus. Rotterdam: Balkema, 1978: 334-9.

9 Caspers RJL, Welvaart K, Verkes J, Hermans J, Leer JWH. The effect of radiotherapy on dysphagia and survival in patients with oesophageal cancer. Radiother Oncol 1988; 12: 15-23.

10 Buset M, des Marez B, Baize M, Bourgeois N, de Boelpaepe C, de Toeuf J, Cremer M. Palliative endoscopic management of obstructive esophagogastric cancer: laser management of obstructive esophagogastric cancer:

11 Krasner N, Barr H, Skidmore C, Morris Al. Palliative laser therapy for malignant dysphagia. Gut 1987; 28: 792-8.

therapy for malignant dysphagia. Gut 1987; 28: 792-8.
12 Ell C, Riemann JF, Lux G, Demling L. Palliative laser treatment of malignant stenoses in the upper gastrointestinal tract. Endoscopy 1986; 18: 21-6.

13 Loizou LA, Rampton D, Atkinson M, Robertson C, Bown SG. A prospective assessment of quality of life after endoscopic intubation and laser therapy for malignant dysphagia. Cancer 1992; 70: 386-91.

14 Rowland CG, Pagliero KM. Intracavity irradiation in the palliation of carcinoma of the oesophagus and cardia. Lancet 1985; ii: 981-2. 
15 Low DE, Pagliero KM. Prospective randomised clinical trial comparing brachytherapy and laser photoablation for palliation of esophageal cancer. $\mathcal{F}$ Thorac Cardiovasc Surg 1992; 104: 173-9.

16 Bader M, Dittler HJ, Ultsch B, Ries G, Siewert JR. Palliative treatment of malignant stenoses of the upper gastrointestinal tract using a combination of laser and afterloading therapy. Endoscopy 1986; 18: 27-31.

17 Nwokolo CU, Payne-James J, Silk DBA, Misiewicz J, Loft DE. Palliation of malignant dysphagia by ethanol induced DE. Palliation of malignant dysphagia by

18 Ogilvie AL, Dronfield MW, Ferguson R, Atkinson M Palliative intubation of the oesophagogastric neoplasms at fibreoptic endoscopy. Gut 1982; 23: 1060-7.

19 Loizou LA, Grigg D, Atkinson M, Robertson B, Bown SG A prospective comparison of laser therapy and intubation in endoscopic palliation for malignant dysphagia. Gastroenterology 1991; 100: 1303-10.

20 Tytgat GNJ, Bartelsman JFWM, Den Hartog Jager FCA Huibregtse $\mathrm{K}$, Mathus-Vliegen $\mathrm{EMH}$. Upper intestinal and biliary tract endoprostheses. Dig Dis Sci 1986; 31: 57S-75S

21 Barr H, Krasner N, Raouf A, Walker RJ. Prospective randomised trial of laser therapy only and laser therapy followed by endoscopic intubation for the palliation of malignant dysphagia. Gut 1990; 31: 252-8.

22 Grundy A. The Strecker esophageal stent in the management of oesophageal strictures: Technique of insertion and early clinical experience. Clin Radiol 1994; 49: 421-4.

23 Knyrim K, Wagner HJ, Bethge N, Keymling M, Vakil N. A controlled trial of an expansile metal stent for palliation of esophageal obstruction due to inoperable cancer. $N$ of esophageal obstruction due

24 Grund KE, Storek D, Becker HD. Highly flexible selfexpanding meshed metal stents for palliation of malignant esophagogastric obstruction. Endoscopy 1995; 27: 486-94.

25 Ell C, May A, Hahn EG. Gianturco-Z stents in the palliative treatment of malignant esophageal obstruction and esophagotracheal fistulas. Endoscopy 1995; 27: 495-500. 